MODELING, IDENTIFICATION AND CONTROL, 1994, VOL. 15, NO. 3, 141-151

doi:10.4173/mic.1994.3.3

\title{
Randomness Reexamined
}

\author{
R. E. KALMAN $\dagger$
}

'Roughly speaking, what we know is science and what we don't know is philosophy.'

Bertrand Russell, ca. $1968^{1}$

\section{A manifesto}

Having become a household name and being supposedly responsible for a huge number of 'applications' of probability to the real world, I may be permitted to say a few words, not necessarily in an unkind way, about the emotional, sociological and philosophical aspects of what in the current fashion is called 'applied probability'.

I see enormous activity ${ }^{2}$, seemingly aimless, I see fanatical devotion to ideas and principles which have grown into a quasi-religion, but not into a scientific discipline; I see younger generation spinning its wheels at problems my contemporaries have not felt worthwhile to devote their lives to. In short, I see a horrible, total, unimaginable mess.

This is a view from the outside. From the inside politics, propaganda and polemics are vigorously and obstinately pursued; science receives lip service only. It is surely going too far to gush about 'the probabilistic revolution' (see Krüger et al. (1986)). Revolution? Indeed. Really? Isn't Alzheimer's disease the right diagnosis?

I would like to comment objectively on this state of affairs. This is not easy because all the discussion is taking place near the boundary separating the two cultures of C. P. Snow - the literary and the scientific. I am in no way schizophrenic and I am not afraid to commute between these two cultures. I see the problem as converting those in the literary culture-more precisely, detoxifying them from their romantic infatuation with probability. To satisfy my friends in the other culture, I shall try to phrase my arguments not only with passion but precision.

It is reassuring to know that $I$ am not the first who felt compelled to comment on the present topic. As a friend with ties to both cultures had put it fifteen years ago, 'the situation you are in is analogous to that which confronted Hercules in being asked to clean out the Augean stables' ${ }^{3}$.

On a politely esoteric level, it is mandatory to quote the late pope of probability, Bruno de Finetti

\section{PROBABILITIES DO NOT EXIST}

(from the prefacc of de Finetti (1974), emphasis by de Finetti.)

Should they? Commingling philosophical ideas of probability with mundane practical or scientific matters (codenamed 'applications') leads to some difficulties.

Received 19 July 1994.

† Swiss Federal Institute of Technology, Zürich, Switzerland and Istituto 'Asinius Pollio'. 
Two of those are especially awkward:

(i) Introducing 'probability' (as here contrasted to 'certainty') into supposed scientific discussions has the effect of rationalizing what we don't know ${ }^{4}$. I don't think this is promising avenue for getting closer to the truth.

(ii) Acting on rumors that probability has become the main theme of prestigious sciences (physics?), less ambitious sciences (economics!) seem to be content to describe the world in probabilistic terms as the best (?) that can be done. This road leads to pseudo-science or, more accurately, ersatz-science. That's not working hard enough.

True, questions of determinacy/indeterminacy have confronted physics for some one hundred years with deep philosophical dilemmas. Yet it is grotesque to claim from just this that physics underwent a probabilistic revolution; this clearly did not happen because probability never became a physical concept. But physics did undergo a Copernican revolution, and to some extent that analogy is relevant, both to this paper and its author.

Has science become revitalized and revolutionized by exploiting probability as the organizing principle? To me this is just mushy and wishful thinking. Historians' claims that this actually happened in, say, economics, or, better, econometrics (see Morgan (1990)), may be relevant for classifying the deluge of literature after 1940. But such talk (so unlike the scientific critique of Copernicus) has contributed nothing to advancing to a higher level of knowledge in science. Econometrics today is hopelessly mired in misunderstandings. Aren't revolutions supposed to create clarity?

The theme of this paper is quite simple. When we research and try to comprehend randomness in Nature we must try to avoid the temptation to automatically, 'explain' randomness in terms of (conventional $=$ games-of-chance $=$ Kolmogorov) probability .

It is our claim that

The majority of observed phenomena of randomness in Nature (always excluding games of chance) cannot be and should not be explained by (conventional) probability theory; there is little or no experimental evidence in favor of (conventional) probability but there is massive, accumulating evidence that explanations and even descriptions should be sought outside of the conventional framework.

\section{Randomness redefined}

This suggests a basic problem: define randomness without probability. Historically, vague notions of randomness were believed to have been made clearer by being translated into probabilistic terminology. (Like random $\rightarrow$ independent random.) Now the task appears to be exactly the opposite: separate randomness from probability.

There is no need to embark on writing a treatise concerning this subtle problem. A simple remark will do. Suppose we consider the sequence of integers

$$
3,7,3,0,9,5,0,4,8,8,0,1,6,8,8 \text {. }
$$

(They happen to be digits 10 through 24 after the decimal point in the decimal expansion of $\sqrt{2 .)}$ Is this sequence random?

The answer depends on what one means (or wants to mean) by 'random'. In the framework of conventional probability theory, the precise question would be: Is this a sequence of (probabilistically) independent random digits?

There is no knowledge vouchsafed to Man in the 20th century that would reveal an unambiguous answer to this question. (If we were given the additional information, 
very hard to deduce from the given digits, that the sequence is part of the decimal expansion of $\sqrt{ } 2$, then the answer would be negative (hence useless); this is so simple because the successive digits of the sequence are generated by an exact, deterministic algorithm and are, therefore, obviously not probabilistically independent.)

There is only one (currently) known way of creating or identifying an independent random sequence. We use an artificial mechanism, not normally found in nature (such as an icosahedral die, a lottery machine, etc.) to generate the sequence by actual experiment. If this is how we got the sequence we have rational grounds to assume that it is 'independent random'. But, alas, there is then (roughly speaking) no known way of assessing how close to the ideal our generating machine comes.

Not being able to test a sequence for 'independent randomness' (without being told how it was generated) is the same thing as accepting that reasoning about an 'independent random sequence' is not operationally useful. Therefore, for me, 'independent randomness' is not a scientific concept. Unfortunately 'independence' has evolved into a such basic and useful assumption in probability theory that is hard to separate 'probability' from 'independence'.

Let us be bold and remove the question of independence, the method of generation and even probability from the way we look at randomness. Then the proper intrinsic definition of randomness becomes obvious. Randomness is the opposite of regularity. In mathematical terms, taking a rather abstract point of view of course, regularity usually appears as uniqueness (as regards the nature of solutions, description of results, etc.). For example, the theorem of Pythagoras shows that a right triangle is not a random object; the features of the right triangle are so regular that from a little bit of information we can (re)construct all we need.

So we are lead to the major claim of this paper:

Definition. Randomness, in situations susceptible to precise mathematical discussion, means nonuniqueness; more generally, a random object is one where nonuniqueness cannot be eliminated even after all regularity relevant to the problem is taken into account. Briefly: a mathematical object is random if and only if it is nonunique modulo all relevant regularities.

Example. A die (a cube).

This is a random object, according to our definition, because the most obvious fact about a die is that it normally comes to rest on some one of its (six) faces. (Similarly, a coin is a random object even if we disregard the possibility that it may come to rest on its (circular) rim.)

The property of nonuniqueness-that it has six faces-is an intrinsic property of a die. By contrast, the 'probability' that the die comes to rest on a given one of its six faces is not an intrinsic property, because (if such a probability exists) it is determined, indirectly, by the system aspects of the situation. (Is the die made of homogeneous material? Does it bounce? Is there a little magnet inside? How it is thrown? What kind of surface does it fall on?) The proper definition of randomness, (ours!), is easy to get from a description of the physical aspects of the situation, while the determination of probabilities involves rather more complex considerations and calculations of the ‘system' type.

This is not to say that the examination of the commonly observed (or believed to be observed) fact that the probabilities of the die falling on any one face are all equal $\left(\frac{1}{6}\right)$ is not interesting. We examine this issue in Section 5. 
Example. A roulette wheel.

The discussion of this example is exactly the same as for the die (randomness involving finitely many alternatives), and again shows that it is best to define randomness without at the same time worrying about probabilities.

Example. The infinite sequence of decimals in the decimal expansion of $\sqrt{ } 2$.

This is a random object in the sense of our new definition. How is this shown? We note that the only (conceivable?) regularity in this particular case occurs when the sequence of decimal digits ultimately (that is, after finitely many digits) becomes periodic. It is known that this is not the case for $\sqrt{ } 2$ because of the theorem, mindlessly taught in the secondary schools, that the decimal expansion of a real number is ultimately periodic if and only if the number is rational; and we know, but of course not by looking at its decimal expansion, that $\sqrt{ } 2$ is not rational, i.e., it is irrational ${ }^{5}$. Of course, it is not $\sqrt{ } 2$ which is a random object in the sense of our new definition but only the decimal expansion of $\sqrt{ } 2$. It is known that the continued fraction expansion of $\sqrt{2}$ is a sequence which ultimately consists of 2's only (clearly not random in any reasonable sense); this follows from of a theorem of Lagrange that is not taught to students in the literary culture.

In the preceding example, randomness is obvious but it is quite unclear what kind of 'probability', if any, may be attached to each occurrence of a decimal digit in the decimal expansion of $\sqrt{ } 2$. Let me hasten to assure the perplexed that all digits are equiprobable (each digit has the probability of $\frac{1}{10}$ ). See Section 5 .

Example. (Rauzy (1976)). Consider the first digit of each of the infinite sequence of decimally noted integers $2^{t}$, where $t$ is a positive integer, enumerated as $t=1,2,3, \ldots$

We claim that this is a random object, that is, a random sequence (in our terminology) of the integers $1, \ldots, 9$ ( 0 does not occur, by definition). That all the digits $1, \ldots, 9$ actually occur is not quite obvious; it takes about 50 terms before 7 and 9 occur. The claim that the sequence is random is based, at first, on a guess about the nonexistence of mathematical results about any kind of regularity of the sequence. (For $\sqrt{ } 2$ this question can be settled using only very elementary number theory.) Somewhat surprisingly we don't even have to worry about 'proving' this guess because, in this interesting special case, we can go much further and actually calculate the probabilities (Rauzy (1976), pages 13-14), as we shall indicate in Section 5.

Again, it is not the family $\left\{2^{t}: t=\right.$ positive integer $\}$ which is a random object, but the family of first digits in the decimal representation of $2^{t}$. If we take the binary representation of $2^{t}$, there is no randomness at all ${ }^{6}$.

Example. The prime numbers.

Since antiquity mathematicians have been fascinated by the sequence of prime numbers, namely $2,3,5,7,11,13, \ldots$ Since (again only roughly speaking), no regularities of this sequence have been discovered in mathematics but only the absence of regularities (for example, it is not possible to generate all the primes by the integers by means of evaluating a fixed polynomial), it is consistent with our definition to say that the totality of primes constitute a random object.

From these examples I conclude that it is not credible to bring back into the discussion the previously examined notion of an (ideal) independent random sequence, for such a notion says nothing useful about our examples, not to speak of the fact that assuming probabilities (that they are equal, for example) bypasses the difficult step of going from a description of randomness to the calculation of probabilities. The hope 
that a revolution will break out by promoting conventional (Kolmogorov) probability theory as a key scientific explanation of what must happen in our examples is-well, a bit naive. It is like taking the shape of the electron (which happens to be unknown) and, by means of powerful axioms dreamt up in a great creative act, telling us all the properties moving electrons must have. Newton was horrified by the intimation that this was how he was doing science, which is why he said, with great contempt, hypothesis non fingo ...

It is perhaps for such reasons that mathematicians do not, as a rule, regard probability as an effective research tool for mathematical puzzles. The usual prejudices are well expressed by the late Russian mathematician Pontryagin by saying 'mathematicians do not believe in probability theory" .

We shall have to comment further on this particular use of the word 'theory'.

\section{The evidence for randomness}

In the last half of the 20th century the evidence for our manifesto,

\section{Distinguish the concept of randomness from that of probability!}

has become overwhelming.

Indeed the discovery of chaos, a collective effort of many ${ }^{8}$, has now evolved into a veritable new paradigm of science, without so to say toppling any other paradigm. Certainly not probability theory (which was never a scientific paradigm anyway) because, by definition, chaos is the study of random (or random-looking) phenomena which are understood to have classical or even quantum-mechanical deterministic causes. The research on chaos, which focuses on randomness in the sense of our definition of the word, never felt a need for grabbing at probabilistic arguments or techniques. Thus, roughly speaking

$$
\text { chaos }=\text { randomness without probability. }
$$

The very issue of determinism vs. indeterminism might as well be left to die. It is less frightening to try to get used to the idea that Nature (at least in some practical, macroscopic sense) does not always prefer uniqueness but can be comfortable with randomness, i.e., nonuniqueness.

A particularly surprising view of randomness, held before the discovery of chaos, may be found in the Nobel lecture of Born (1954). He uses expressions like 'when determinacy lapses into indeterminacy' without any logical, mathematical, empirical, let alone physical, argumentation to back up such bizarre imagery. Even more shockingly, there are rumors about a current investigation (by physicists?) of the trajectory of a die, between the point where it is tossed out of the point where it comes to rest, the aim being to determine where, along the trajectory, determinacy has lapsed into indeterminacy. Since the randomness of the die, according to our new definition, is obvious and physically trivial, while probabilities arise in a complicated way, our position is that there is no meaning, operational, physical or philosophical, to be attached to 'when determinacy lapses into indeterminacy.'

One wonders how contemporary physicists can hope to avoid the great Newton's wrath, who thundered, referring to such and many other funny ways of doing (?) physics, that these ways 'prejudicia sunt et scientiam non pariunt."9. 


\section{Is econometrics a paradigm for probability?}

Let us hope that potential damage inflicted on the world of physics by Born and his (younger?) disciple has been minimized already by the cruel device of denying tenure to the latter. But the situation in econometrics is not quite so simple.

Although loud and opposing voices were raised, especially by Frisch (1934), to the gradually prevailing dogma of imposing probability and then statistics on the treatment of noisy data - we may call this, roughly speaking, the Fisherian paradigm of sampling and estimation (Fisher: 'the world is a parametrized family of probability distributions') - somehow the addictiveness of the idea of probability resulted in weeding out the scientists from among the econometricians. Is the damage only temporary?

Typical commentary, in this context, may be found in Hendry and Morgan (1989). They respectfully recall Frisch's ideas (undoubtedly rough and sketchy), emphasize Frisch's independence of the orthodoxy of Fisher (a historical fact), but then rather high-handedly transcribe Frisch's attempts into the language of Fisher and so arrive at showing, easily, that Frisch did not know what he was doing. See Kalman (1994) for comments on this critique.

The Fisher-Haavelmo paradigm in econometrics developed after 1940 (see Morgan (1990) for accurate historical background but sterilized of scientific critique) postulates that data in econometrics must be explained by means of a probabilistic model. (So this paradigm started life as religion and not as science.) Frisch, working about a decade earlier, was consciously attempting to avoid this prejudice, and tried to concentrate on the direct scientific (or, as I would say today, system-theoretic) problem of identifying (a model of) economic relations from economic data. Even by hindsight Hendry and Morgan are rather skeptical about this enterprise ${ }^{10}$, because Frisch-who constructed a simulation experiment to generate data ('constructed data') according to the rules of a Fisherian sampling model-refused to analyze the data he so obtained according to the prejudices of Fisher (the probabilistic dogma), which is why Frisch succeeded fairly well with his independent identification method.

This comedy of errors came to an end on July 20, 1983 at Sophia Antipolis (France). In effect, this was also the end of the 'probabilistic revolution' (if it ever existed) in econometrics. The event is precisely datable. In the course of computing experiments conducted by the writer on real economic data (taken from the textbook of Malinvaud (1978), which was composed in the spirit of the orthodox Fisher-Haavelmo paradigm) a major discovery was made. The experiments were intended to check the nonprobabilistic theory of identification of Frisch. This theory was immediately refuted by the results of the experiments, and, by luck, the computations showed much more-indeed an experimental discovery!

What killed Haavelmo's hope that econometric models must be based on probability? Simply the confrontation with data. Computations in July 1983 have shown (and since 1983 repeatedly confirmed) that the fundamental axiom of the probability approach to econometrics - that noise is probabilistic - is not compatible with the data; certain events whose probability should be negligible are nonetheless frequently observed. In the terminology of Popper, this falsifies the probabilistic paradigm. The net results is that identification is more difficult than if probability were the true basis of econometrics, but this does not necessary mean that economic data is so noisy that it cannot be used for accurate identification.

If (mainstream) econometrics had died already in 1983, how do we explain the orgy of self-congratulation and celebration (probabilistic revolution) still going on today? 
The human mind is infinitely fascinated by and protective of the products of its own imagination. In the sciences, this is known as 'artifacts'; sadly, even scientific paradigms seem to be destined to decay because they tend to forget the problems that engendered them while continuing to study the artifacts created within the paradigm by the paradigm itself. It is sobering to recall Fliess's remark about his friend Freud: 'Der Gedankenleser liest in den Anderen nur seine eigenen Gedanken' ('The mind reader reads in the minds of others only his own thoughts'.).

So it is really very interesting that the arguments in Haavelmo (1944) in favor of probability appear to be unimpeachable when they are reinterpreted as being in favor of randomness (as I have defined it here). But the jump-made unconsciously by Haavelmo-from randomness to probability was a gigantic non sequitur,

Is this excusable? I think, yes, for Haavelmo, but not for competent historians of science. All working scientists know about such things. To the average research worker, it is the 'not invented here' syndrome. Or: 'if I haven't thought of it, it does not exist.'

Historically speaking, the same mistake occurred at the beginning of the 19th century when it was taken for granted that Euclid's geometry has to be the correct geometry for the physical world, simply because it was then the only known geometry. With the discovery of the noneuclidean geometries, this argument for the inevitability of Euclidean geometry in the real world became a nonissue. And this is not really excusable. After all, a hundred years before (1713) Newton was already on record against prejudice. Was there any argument for

$$
\text { Euclidean geometry }=\text { Nature's geometry }
$$

other than a prejudice?

Today, there is a lot of evidence that there may well be many different kind of probabilities (Accardi (1994)). Haavelmo's error that

$$
\text { randomness }=(\text { conventional }) \text { probability }
$$

is just another example of scientific prejudice. Historians of science know, of course, that Copernicus's great contribution-the creative act that engendered a Western science independent of as well as superior to the Greeks--was to point out, by implacable logic, that the classical evidence in favor of the geocentric view did not consist of scientific facts, after all, but only of scientific prejudices.

\section{The origin of probabilities}

The (noncontroversial) remark is often heard that the 'theory of probabilities', as known and taught at the end of the 20th Century, is not a theory since it deals with probabilities as given and does not comment on how these probabilities are explained, measured, induced from other information, etc. It would be less confusing if the old-fashioned terminology 'calculus of probabilities' were brought back into current usage.

We pause briefly to look at the real theoretical question of how probabilities arise, to further illuminate the sharp conceptual distinction - that we insist on-between randomness and probabilities.

Here is perhaps the oldest problem on which definite results are available.

Consider the unit interval $[0,1)$ on the real line. Its points are real numbers. Since, according to contemporary mathematics, nothing more can be said, we regard $[0,1)$ a random object in strict accordance with our new definition of randomness. 
The question arises, is there a probability distribution to be 'naturally' attached to this random object? For example, naive statistics or fuzzy thinking would say that (i) the distribution might as well be assumed to be uniform (height 1); (ii) if we take points 'randomly' from the real line we are sampling just this distribution.

This is much too vague. However, there is a well known (and completely precise) mathematical theory relevant to the situation, which evolved around 1909-1916, culminating in the famous

\section{Gleichverteilungssatz of Weyl (1916). Consider the family of numbers}

$$
\{\alpha t \bmod 1: t=1,2,3, \ldots, T\} .
$$

The elements of this family are uniformly distributed on $[0,1)$ (in the sense that if $J \subset[0,1)$ is any subinterval, the number of elements falling into $J$ is given by the number (length $J$ ). $T$ in the limit as $T \rightarrow \infty$ ) if and only if $\alpha=$ irrational.

This is a most remarkable mathematical result because it connects the pure mathematical notion of an irrational number to something related to probability, about which we have already speculated ${ }^{\prime \prime}$.

Indeed, if we call the number $\alpha t$ mod 1 (where $\alpha=$ fixed) the 't-th sample taken from the unit interval' the theorem can be viewed as describing a scheme for sampling a uniform distribution over the unit interval. But caution: the 'distribution' is a gedanken-object; it is created simply by the claim and reinterpretation of the theorem, it does not exist in any natural sense over $[0,1)$. We could assume it to exist, by fiat, but then we leave mathematics and enter probability theory. The number $T$ can be interpreted as 'sample size'. (Many results, found since 1916, describe the 'sampling distribution' of the family for finite $T$, not just in the limit $T \rightarrow \infty$.)

So we might be tempted to think of Weyl's theorem as a theoretical basis for statistics and hence for econometrics. This is, perhaps unfortunately, not so, because

(i) the sampling process described by $\{\alpha t \bmod 1, \alpha=$ fixed irrational $\}$ is not independent in $t$ (obviously), but classical statistical sampling theory is. Statistical sampling theory can be justified by assumption (prejudice) but not by Weyl's theorem.

The word 'independent sampling' removes classical statistical sampling theory from any strict logical relation with mathematics and mathematical physics, at least as far as the latter subjects are developed today, because

(ii) no mathematical results are known that would generalize Weyl's theorem in the direction of independent sampling.

Sampling, however, is just one interpretation of Weyl's theorem. We can also look at it as providing a machinery for calculating probabilities of certain random objects, where 'probability' is to be thought of in the frequentist interpretation, namely the number of events (say, the value of a digit) relative to the total sample size $T$. An interesting application is Rauzy's example. In the limit as $T \rightarrow \infty$ the probability (frequency) of the first decimal digit $k_{t}$ of $2^{t}$ is given by the exact formula

$$
\log _{10}(k+1)-\log _{10} k \quad(k=1, \ldots, 9)
$$

(see Rauzy [1976, page 13]). Note that the sum over $k$ is 1 . This is not a uniform distribution and it is quite nonobvious without calculations. Yet these probabilities are logically impeccable as a rigorous consequence of a hard mathematical result. 
(The reader might check and verify excellent agreement with the formula for sample sizes of one hundred or more.)

If it were possible to extend Weyl's theorem to the special sequence $\left\{10^{t} \sqrt{ } 2\right.$ mod 1 ) we would be sure that the digits in the decimal expansion of $\sqrt{ } 2$ are uniformly distributed. (They certainly are, at least on the evidence of the first 50000 digits.) But this has turned out to be an extremely difficult technical mathematical problem which is still unsolved today.

A further (successful) mathematical extension of Weyl's theorem leads to showing, quite generally, that in finite situations (for example, finite number of distinct events) it is natural to define probabilities as uniform. Thus, at least in the special case of both the die and the roulette wheel, the traditional assumption of equal probabilities can be bolstered by considerable mathematical evidence (nothing being said about independence).

Both the die and the roulette wheel are successfully 'explained' in the old theory (probability is an axiom) and in the new theory (randomness exists irrespective of probability). If the issue were axiomatic probability (and not randomness), and if probability were taken as a research problem in modern physics, there would be a mad rush to detect, identify, measure, distill, purify, synthesize, etc. little roulette wheels, dice, and whatnot in the constituents of matter, protons, electrons, quarks, whatever. In this sense, probability could do for physics what genetics (DNA) did for biology. It speaks well for the common-sense of experimental physics that such a mad rush has not (yet?) materialized. In any event, no roulette wheels have been found inside the atom. Hence extrapolating roulette-wheel probability at Nature is a highly dubious proposition; it is better to look at randomness with a non-roulette-wheel-bound mind. This, for me, provides adequate evidence to be in comfortable agreement with Einstein that 'God does not play dice.'

\section{Summary}

Randomness is a fact of the real world and thus of scientific interest, as are all facts which are not self-evident. Probability is an intellectual construct. It does not exist in the real world. It is not something quantifiable, measurable, concrete. It is not of scientific concern today because it exists only in the self-interest of gamblers or the imagination of statisticians or the mind-reading of philosophers.

There was and is no such thing as a probabilistic revolution in science although there does seem to be something going on. I hope it is not a social Alzheimer's. It is the ultimate hypotheses fingo. Obnoxious as nerds undoubtedly are, should we not put up with a nerd (even a geek?) like Newton and adopt his motto, hypotheses NON fingo? He got closer to the truth that way and I think that we will, some day, too.

\section{Notes}

1. This opinion of Bertrand Russell was kindly communicated to me by Dr. Hassan Mortazavian together with a tape recording of an interview where Russell himself can be heard to utter these words. The quotation was intended to be heading for Mortazavian's paper in Machlup and Mansfield (1988, p. 517-545), but it was deleted by fiat of a dictatorial editor, the late Fritz Machlup.

2. Kalman filtering was first publicly presented (to somewhat more than polite applause) on April 1, 1959. Twenty-five years later it was estimated that about 100000 papers, technical reports, books, expository articles, summer courses, etc. etc. have appeared concerned with applications of Kalman filtering theory. At present a rough estimate might be 200000 . But please note: Kalman filtering is not a triumph of applied probability; the theory has only 
a slight inheritance from probability theory while it has become an important pillar of system theory.

3. This is well known as the sixth labor of Hercules. It is also well known that the person who suggested the problem to Hercules was not happy with the latter's chosen method of solution, and that this disagreement had serious consequences for the former. We see here (attention scientists) the almost magical relevance of Greek literary culture to the sociology of our own times.

4. Until recent times, the question of who is the father could not be scientifically assessed and one tried to rely on indirect evidence. To the best of my knowledge probability theory was never applied in such cases to reconstruct the missing or uncertain information.

5. The Greeks thought that something regular is 'rational' and that the opposite is 'irrational'. These words have somehow found their way into contemporary mathematical terminology. From our point of view it would be quite logical to define 'random' as 'nonrational'. However, this would be giving a bit too much credit to the Greeks who did not explore the proposition random $=$ nonrational, did not explore this idea, did not develop probability theory etc. Yet it is amazing how many of the difficult unsolved problems of modern mathematics (primes, Riemann hypothesis, Fermat's last theorem, etc.) are somehow tied up with the random, that is, the nonrational, that is, something that is not readily accessible to the rational thinking that mathematicians are proud of.

6. The insight that randomness is related to the special ways in which objects (integers, a language) are coded into mathematical format is perhaps the main contribution of Shannon to what is called since then information theory; sadly, this is not the point of view that the historians of the 'probabilistic revolution' have focused on.

7. Quoted in Kalman (1985, page 29).

8. The phenomenon of chaos was independently discovered (the word was not then current) in the course of the writer's Master of Science dissertation (Kalman (1954)), as a by-product of the study of certain examples of nonlinear difference equations. This was elaborated on in a later paper (Kalman (1956)), but without consciously formulating the basic problem, namely, that probability theory cannot be (probably?) combined with other mathematical structures since the axioms of probability are so to say incommensurate with anything else. Hence the dilemma of describing random phenomena resulting from deterministic mathematical structures - which is what gave rise to chaos research. Interestingly, similar observations and investigations were made by many at the time; for example, joint work (unpublished) by J. von Neumann and S. Ulam around 1950.

9. Although there exist (at least) two autographs from Newton concerning this phrase (without corrections, unusual for him), these were not published until recently. The phrase was created around 1713 by Newton in the course of the stylistic exercise of composing the concluding Scholium Generale for the second edition of the Principia. He ended up by overcondensing some sentences, including the quoted one, into just three words, hypotheses no-fingo. See Cohen (1978, page 243).

10. They write (Hendry and Morgan (1989, p. 39, middle)) that 'model selection (under Frisch's ground rules) looks hopeless'.

11. See note 5 .

\section{REFERENCES}

ACCARDI, L. (1994). Personal communications, based on many years of study to discover and elucidate the precise nature of 'chance' in quantum mechanics.

BORN, M. (1954). Nobel lecture, published in Les Prix Nobel, 1954, Nobel Foundation, Stockholm.

COHEN, I. B. (1978). Introduction to Newton's Principia, Harvard University Press, 380 pages. DE FINETTI, B. (1974). Theory of probability; a critical introductory treatment, Wiley, 2 volumes, 300 and 375 pages.

FrISCH, R. 1934. Statistical Confluence Analysis by Complete Regression Systems, Publication No. 5 of Econometric Institute, University of Oslo, Norway, 192 pages.

HAAVElmo, T. (1944). 'The probability approach to econometrics', Econometrica, 12 (Supplement).

HENDRY, D. F. and Morgan, M. S. (1989). 'A re-analysis of confluence analysis', Oxford Economic Papers, 41: 35-52. 
KaLman, R. E. (1954). 'Phase-plane analysis of nonlinear sampled-data servomechanisms', M. S. dissertation, MIT, 68 pages.

Kalman, R. E. (1956). 'Nonlinear aspects of sampled-data control systems', article in Proc. Symposium on Nonlinear Circuit Analysis, edited by J. Fox. Polytechnic Institute of Brooklyn, pages 273--313.

Kalman, R. E. (1985). 'Identification of noisy systems' (in Russian), Uspekhi Mat. Nauk., 40: 29-37.

KaLmAN, R. E. (1993). Nine Lectures on Identification, Springer lecture Notes on Economics and Mathematical Systems.

KALMAN, R. E. (1994). 'Identification in econometrics as a system-theoretic problem', to appear. KrÜGER, L., Gigerenzer, G. and Morgan, M. S. (editors) (1987). The Probabilistic Revolution. Volume 2: Ideas in the Sciences (book, various articles), MIT Press, 459 pages.

MACHLup, F. and MANSfield, U. (editors) (1983). The Study of Information: Interdisciplinary Messages (book, various contributors), Wiley, 743 pages.

Malinvaud, E. (1978) Méthodes Statistiques de l'Économetrie, 3ème édition, Dunod, Paris, 846 pages.

Morgan, M. S. (1990). The History of Econometric Ideas, Cambridge University Press, 296 pages.

Rauzy, G. (1976). Propriétés Statistiques de Suites Arithmétiques, Presses Universitaires de France, 133 pages.

WEYL, H. (1916). 'Über die Gleichverteilung von Zahlen mod. Eins', Mathematische Annalen, 77: 313-352. 